\title{
A constructive approach to accessible group classes
}

\author{
Francesco de Giovanni ${ }^{1} \cdot$ Marco Trombetti $^{1}$
}

Received: 16 April 2021 / Accepted: 16 July 2021 / Published online: 3 August 2021

(C) The Author(s) 2021

\begin{abstract}
Let $\mathfrak{X}$ be a group class. A group $G$ is an opponent of $\mathfrak{X}$ if it is not an $\mathfrak{X}$-group, but all its proper subgroups belong to $\mathfrak{X}$. Of course, every opponent of $\mathfrak{X}$ is a cohopfian group and the aim of this paper is to describe the smallest group class containing $\mathfrak{X}$ and admitting no such a kind of cohopfian groups.
\end{abstract}

Keywords Accessible group class $\cdot$ Accessible closure $\cdot$ Cohopfian group

Mathematics Subject Classification $20 \mathrm{E} 34 \cdot 20 \mathrm{~F} 22$

\section{Introduction}

Let $\mathfrak{X}$ be a class of groups. A non-trivial group is said to be minimal non- $\mathfrak{X}$ if it is not an $\mathfrak{X}$ -group, but all its proper subgroups belong to $\mathfrak{X}$; minimal non- $\mathfrak{X}$ groups are also called opponents of $\mathfrak{X}$. We say that $\mathfrak{X}$ is accessible if every locally graded group whose proper subgroups belong to $\mathfrak{X}$ is either finite or an $\mathfrak{X}$-group, i.e. if any locally graded opponent of $\mathfrak{X}$ is finite. Recall here that a group is locally graded if all its finitely generated non-trivial subgroups have proper subgroups of finite index. In particular, if $\mathfrak{X}$ contains the class $\mathfrak{F}$ of all finite groups, then it is accessible if and only if there are no opponents of $\mathfrak{X}$ within the universe of locally graded groups. A group class which is not accessible is called inaccessible, so a group class is inaccessible if and only if it admits an infinite locally graded opponent.

It is easy to show that the class $\mathfrak{U}$ of abelian groups is accessible and $\mathfrak{U}$ shares such a property with other relevant classes of groups, like, for instance, the class $\mathfrak{N}_{c}$ of nilpotent

In memoriam Carlo Casolo.

The authors are supported by GNSAGA (INdAM) and are members of AGTA-Advances in Group Theory and Applications (www.advgrouptheory.com)

Francesco de Giovanni

degiovan@unina.it

Marco Trombetti

marco.trombetti@unina.it

1 Dipartimento di Matematica e Applicazioni, Università degli Studi di Napoli Federico II, Napoli, Italy 
groups of class at most $c$ and the class $\mathfrak{S}_{d}$ of soluble groups of derived length at most $d$ (see [3] and [6], respectively). On the other hand, the consideration of the locally dihedral 2-group shows that the class $\mathfrak{N}$ of nilpotent groups is inaccessible, while it seems to be an open question whether the class $\mathfrak{S}$ of soluble groups is accessible or inaccessible.

Inaccessible group classes are closely related to the important concept of a cohopfian group. Recall that a group $G$ is cohopfian if it is not isomorphic to any of its proper subgroups, or equivalently if every injective endomorphism of $G$ is an automorphism. All finite groups are obviously cohopfian and an infinite locally graded group is cohopfian if and only if it is an opponent of a suitable inaccessible group class. We refer to the recent paper [16] for an extensive analysis of this topic.

The aim of this paper is to provide a further contribution to the study of inaccessible group classes and consequently to the knowledge of cohopfian groups. In particular, for any group class $\mathfrak{X}$, we describe the smallest accessible group class $\mathbf{A}(\mathfrak{X})$ containing $\mathfrak{X}$. Of course, all groups in $\mathbf{A}(\mathfrak{X}) \backslash \mathfrak{X}$ are cohopfian. Actually, $\mathbf{A}(\mathfrak{X})$ is constructed as the union of an ascending chain of group classes $\mathbf{A}_{\alpha}(\mathfrak{X})$ containing $\mathfrak{X}$ and as we climb up the chain, we find cohopfian groups which are richer in cohopfian subgroups. The final section of the paper contains a number of concrete examples of accessible closures.

Our notation is mostly standard and can be found in [28]. Notice also that some of our arguments concerning classes may be precisely developed at least in the Morse-Kelley set theory (see [25]).

\section{Accessible group classes}

The aim of this section is to describe some general properties of accessibility, with special attention to the relationship between accessible and local group classes. In addition to this, we produce many examples of accessible classes that will be used in Sect. 4 to determine certain interesting accessible closures.

It is quite obvious that the intersection of any collection of accessible group classes is likewise accessible. Actually, for the intersection of two accessible group classes, this fact is a special case of the following easy lemma.

Lemma 2.1 Let $\mathfrak{X}$ and $\mathfrak{Y}$ be group classes. If $\mathfrak{Y}$ is accessible and does not contain infinite locally graded opponents of $\mathfrak{X}$, then the intersection $\mathfrak{X} \cap \mathfrak{Y}$ is accessible.

Proof Let $G$ be an infinite locally graded group whose proper subgroups belong to $\mathfrak{X} \cap \mathfrak{Y}$. Since $\mathfrak{Y}$ is accessible, $G$ is a $\mathfrak{Y}$-group. Then $G$ cannot be an opponent of $\mathfrak{X}$, so it is also an $\mathfrak{X}$-group. Therefore $G$ is in $\mathfrak{X} \cap \mathfrak{Y}$ and hence $\mathfrak{X} \cap \mathfrak{Y}$ is accessible.

Since the class of torsion-free groups is obviously accessible, it follows from Lemma 2.1 that if $\mathfrak{X}$ is a group class whose locally graded opponents are periodic, then the class of all torsion-free $\mathfrak{X}$-groups is accessible. Further into the text, we will exhibit examples of this situation.

In contrast to the above consideration, it turns out that the union of accessible group classes need not be in general accessible; this is, for instance, the case for the class of nilpotent groups, as 


$$
\mathfrak{N}=\bigcup_{c \in \mathbb{N}} \mathfrak{N}_{c}
$$

In order to prove that even the union of two accessible group classes need not be accessible, some more work is needed.

Let $\pi$ be a proper non-empty subset of the set of all prime numbers $\mathbb{P}$, and let $\mathfrak{D}_{\pi}$ be the class of all torsion-free locally cyclic groups in which every non-trivial element has finite $p$-height for some $p \in \pi$; of course, $\mathfrak{D}_{\pi}$ is subgroup closed. Assume for a contradiction that $\mathfrak{D}_{\pi}$ admits an infinite locally graded opponent $G$. It is easy to see that $G$ is isomorphic to a subgroup of the additive group $\mathbb{Q}$ of all rational numbers and $G \not \mathbb{Q}$ since $\pi \neq \mathbb{P}$. Thus

$$
G \simeq G^{m}<G
$$

for some positive integer $m$, which is obviously impossible. Therefore the group class $\mathfrak{D}_{\pi}$ is accessible. Moreover, since $\pi^{\prime}=\mathbb{P} \backslash \pi$ is a proper non-empty subset of $\mathbb{P}$ too, we can analogously consider the accessible group class $\mathfrak{D}_{\pi^{\prime}}$. On the other hand, the group class

$$
\mathfrak{D}_{\pi} \cup \mathfrak{D}_{\pi^{\prime}}
$$

consists of all groups isomorphic to a proper subgroup of $\mathbb{Q}$ and hence it is inaccessible.

Although the classes $\mathfrak{D}_{\pi}$ and $\mathfrak{D}_{\pi^{\prime}}$ in the above example are accessible, they are not local. Recall here that a group class $\mathfrak{X}$ is said to be local if it contains every group $G$ such that each finite subset of $G$ lies in an $\mathfrak{X}$-subgroup; in particular, if the class $\mathfrak{X}$ is subgroup closed, then $\mathfrak{X}$ is local if and only if it contains all groups whose finitely generated subgroups are $\mathfrak{X}$-groups. Actually, it turns out that local group classes are not in general accessible, even if they are subgroup closed. In fact, consider a finitely generated torsion-free nilpotent group $W$ which is cohopfian (for the existence of such groups see, for instance, the proof of Proposition 4 in [33]), and let $\mathfrak{W}$ be the class consisting of all groups in which every finitely generated subgroup is isomorphic to a proper subgroup of $W$. Then $\mathfrak{B}$ is a local class and $W$ is an opponent of $\mathfrak{W}$, so that the class $\mathfrak{W}$ is inaccessible.

Our next example shows that the union of two accessible group classes need not be accessible even within the universe of periodic groups.

Let $p$ and $q$ be distinct prime numbers and let $\mathfrak{A}(p, q)$ be the class consisting of the trivial groups and of all abelian groups that can be decomposed into the direct product of a non-trivial locally cyclic $p$-group and a cyclic $q$-group. It is straightforward to show that $\mathfrak{A}(p, q)$ is an accessible class. On the other hand, the class

$$
\mathfrak{A}(p, q) \cup \mathfrak{A}(q, p)
$$

is not accessible, admitting the direct product $C=C_{p^{\infty}} \times C_{q^{\infty}}$ as an opponent. Here, again the consideration of the group $C$ shows also that the class $\mathfrak{A}(p, q)$ is not local, for any choice of the primes $p$ and $q$.

Let $\mathfrak{X}$ be a local group class. Obviously, $\mathfrak{X}$ admits only finitely generated opponents and in particular the locally graded ones belong to the class $\mathfrak{X} \mathfrak{F}$, i.e. they contain a normal $\mathfrak{X}$ -subgroup of finite index (this is, for instance, the case of the class $\mathfrak{W}$ considered above). It follows that $\mathfrak{X}$ is accessible, provided that $\mathfrak{X}=\mathfrak{X} \mathfrak{F}$. On the other hand, if we assume that $\mathfrak{X}$ is accessible, it may happen that $\mathfrak{X} \mathfrak{F}$ is inaccessible, even if it is subgroup closed (see, for instance, [15]).

Our next statement shows in particular that the class of groups which are locally $\mathfrak{X} \mathfrak{F}$ is accessible whenever the group class $\mathfrak{X}$ is subgroup closed. The smallest local group class 
containing $\mathfrak{X}$ is usually denoted by $\mathbf{L} \mathfrak{X}$; thus $\mathbf{L} \mathfrak{X}$ is the class of all groups in which every finitely generated subgroup lies in some $\mathfrak{X}$-subgroup (or it is actually an $\mathfrak{X}$-subgroup if $\mathfrak{X}$ is subgroup closed). Notice also that all finitely generated groups in the class $\mathbf{L} \mathfrak{X}$ are $\mathfrak{X}$ -groups.

Lemma 2.2 Let $\mathfrak{X}$ be a group class which is closed with respect to normal subgroups of finite index. Then the class $\mathbf{L}(\mathfrak{X} \mathfrak{F})$ is accessible.

Proof Assume for a contradiction that $\mathbf{L}(\mathfrak{X} \mathfrak{F})$ admits an infinite locally graded opponent $G$. Then $G$ must be finitely generated and so it contains a proper subgroup $H$ of finite index. Clearly, $H$ is finitely generated and hence it contains an $\mathfrak{X}$-subgroup $K$ of finite index. Since $\mathfrak{X}$ is closed with respect to normal subgroups of finite index, it follows that the normal core $K_{G}$ is an $\mathfrak{X}$-subgroup of finite index of $G$, a contradiction.

It follows in particular from the above result that the classes $\mathbf{L}(\mathfrak{S} \mathfrak{F})$ of all locally (soluble-by-finite) groups and $\mathbf{L}(\mathfrak{N} \mathfrak{F})$ of all locally (nilpotent-by-finite) groups are accessible. We also remark that in the statement the class $\mathbf{L}(\mathfrak{X} \mathfrak{F})$ cannot be replaced by $(\mathbf{L} \mathfrak{X}) \mathfrak{F}$, even when $\mathfrak{X}$ is local and accessible, as the choice $\mathfrak{X}=\mathfrak{A}=\mathbf{L} \mathfrak{A}$ shows (see, for instance, [2]).

Lemma 2.3 Let $\mathfrak{X}$ be a group class which is contained in the class Max of all groups satisfying the maximal condition on subgroups. If all infinite locally graded opponents of $\mathfrak{X}$ are not finitely generated, then the class $\mathbf{L} \mathfrak{X}$ is accessible.

Proof Let $G$ be any locally graded opponent of $\mathbf{L} \mathfrak{X}$. Then $G$ is finitely generated and so it contains a proper subgroup $X$ of finite index. Clearly, $X$ is likewise finitely generated and hence it is an $\mathfrak{X}$-group. It follows that $G$ satisfies the maximal condition on subgroups. Thus all proper subgroups of $G$ belong to $\mathfrak{X}$ and so $G$ is an opponent of $\mathfrak{X}$. Therefore $G$ is finite and the class $\mathbf{L} \mathfrak{X}$ is accessible.

Of course, Lemma 2.3 applies to any accessible class contained in the class Max. Although the class Max is clearly inaccessible, its infinite locally graded opponents cannot be finitely generated, so Lemma 2.3 also yields that $\mathbf{L}(\operatorname{Max})$ is accessible. We point out that, dually, if $\mathfrak{X}$ is any subclass of the class Min of all groups with the minimal condition on subgroups, then $\mathbf{L} \mathfrak{X}$ is an accessible class.

It should also be remarked that there exist subgroup closed subclasses of Max to which Lemma 2.3 does not apply, like, for instance, the intersection $\mathfrak{W} \cap \operatorname{Max}$, where $\mathfrak{W}$ is the local class considered above. On the other hand, some of the most important subclasses of Max admit the Prüfer groups as the only infinite locally graded opponents. In fact, this is the case of the class $\mathfrak{P}$ of polycyclic groups (see [12], Corollary 2.6) and the next result shows that the same conclusion holds for the class $\mathbf{P}_{n}^{\mathscr{C}}$ of supersoluble groups and for the class of finitely generated nilpotent groups.

Let $\mathfrak{U}$ be a class of groups. A group class $\mathfrak{X}$ is called a $\mathfrak{U}$-Fitting class if $\mathfrak{X} \leq \mathfrak{U}$ and $\mathfrak{X}$ contains every $\mathfrak{U}$-group which is the product of two normal $\mathfrak{X}$-subgroups. If $\mathfrak{U}$ is the class of all groups, $\mathfrak{U}$-Fitting classes are precisely the usual Fitting classes. Notice also that every group class $\mathfrak{X}$ is trivially $\mathfrak{X}$-Fitting.

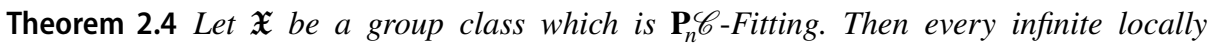
graded opponent of $\mathfrak{X}$ is a Prüfer group. 
Proof We first prove the statement for the class $\mathbf{P}_{n} \mathscr{C}$. Let $G$ be an infinite locally graded opponent of $\mathbf{P}_{n} \mathscr{C}$ and assume for a contradiction that $G$ is polycyclic. Then $G$ contains a normal subgroup of finite index which is poly-(infinite cyclic), and hence it admits a finite homomorphic image $G / M$ whose order is divisible by at least four prime numbers. If $N$ is any normal subgroup of finite index of $G$, the factor group $G / M \cap N$ cannot be minimal non-supersoluble (see [10]) and so it must be supersoluble. Therefore all finite homomorphic images of $G$ are supersoluble and hence $G$ itself is supersoluble (see [1]), a contradiction. Thus $G$ is not polycyclic and so it is a group of type $p^{\infty}$ for some prime $p$ (see [12], Corollary 2.6).

Consider now an arbitrary $\mathbf{P}_{n} \mathscr{C}$-Fitting group class $\mathfrak{X}$ and let $G$ be an infinite locally graded opponent of $\mathfrak{X}$ which is not a Prüfer group. Since $\mathfrak{X} \leq \mathbf{P}_{n}^{\mathscr{C}}$, it follows from the first part of the proof that $G$ is supersoluble, so it admits an infinite homomorphic image which is either cyclic or dihedral. Thus $G$ is the product of two proper normal subgroups and hence it belongs to $\mathfrak{X}$, a contradiction.

It follows now from Lemma 2.1 that the class of polycyclic torsion-free groups, the class of supersoluble torsion-free groups and the class of finitely generated nilpotent torsion-free groups are accessible.

The consideration of the famous example of Heineken and Mohamed [22] shows that there exist infinite locally graded opponents of the class $\mathfrak{N}$ of nilpotent groups having a complicated structure. On the other hand, it is known that locally nilpotent groups form an accessible class (see [13]); in particular, all infinite locally graded opponents of $\mathfrak{N}$ are locally nilpotent and so they are even primary groups (see, for instance, [7], Theorem A). Thus also the class of torsion-free nilpotent groups is accessible by Lemma 2.1. It is also known that the class of hypercentral groups and the class of hypercyclic groups have the same infinite locally graded opponents and these are periodic (see [13]), so again Lemma 2.1 yields that the class of torsion-free hypercentral groups and the class of torsion-free hypercyclic groups are accessible.

Locally supersoluble groups form an accessible class (see [13]). Moreover, since Prüfer groups are the only infinite locally graded opponents of the class of polycyclic groups, Lemma 2.3 has the following interesting consequence.

\section{Corollary 2.5 The class $\mathbf{L} \mathfrak{P}$ is accessible.}

It follows from Lemma 2.2 that the class $\mathbf{L}(\mathfrak{P F})$ of all locally (polycyclic-by-finite) groups is accessible, and our next results show that this property holds for many natural subclasses of $\mathbf{L}(\mathfrak{P} \mathfrak{F})$.

Lemma 2.6 Let $\mathfrak{X}$ and $\mathfrak{Y}$ be subgroup closed group classes consisting of polycyclic-byfinite groups and such that $\mathfrak{X} \cap \mathfrak{Y} \leq \mathfrak{F}$. Then the infinite locally graded opponents of $\mathbf{L} \mathfrak{X} \cup \mathbf{L} \mathfrak{Y}$ ) are finitely generated opponents either of $\mathfrak{X}$ or of $\mathfrak{Y}$.

Proof Let $G$ be an infinite locally graded opponent of the local class $\mathbf{L} \mathfrak{X} \cup \mathbf{L} \mathfrak{Y}$. Then $G$ must be finitely generated and so it contains a proper normal subgroup $N$ of finite index. Obviously, $N$ is finitely generated, so it belongs either to $\mathfrak{X}$ or to $\mathfrak{Y}$ and hence it is polycyclic-by-finite. Thus $G$ itself is polycyclic-by-finite. Suppose that $G$ contains proper subgroups of finite index $X$ and $Y$ such that $X \in \mathfrak{X}$ and $Y \in \mathfrak{V}$. Since $\mathfrak{X}$ and $\mathfrak{Y}$ are subgroup closed, the intersection $X \cap Y$ belongs to $\mathfrak{X} \cap \mathfrak{V}$ and so is finite, a contradiction. Therefore 
either all proper subgroups of finite index of $G$ are in $\mathfrak{X}$ or all are in $\mathfrak{Y}$. Since each proper subgroup of $G$ is contained in a proper subgroup of finite index, it follows that $G$ is either an opponent of $\mathfrak{X}$ or of $\mathfrak{Y}$. The proof is complete.

The above statement has a number of interesting consequences.

Corollary 2.7 Let $\mathfrak{X}$ and $\mathfrak{Y}$ be subgroup closed group classes consisting of polycyclic-byfinite groups and such that $\mathfrak{X} \cap \mathfrak{Y} \leq \mathfrak{F}$ and let $G$ be an opponent of $\mathfrak{X} \cup \mathfrak{Y}$ which satisfies the maximal condition on subgroups. Then $G$ is either an opponent of $\mathfrak{X}$ or of $\mathfrak{Y}$.

Corollary 2.8 Let $\mathfrak{X}$ and $\mathfrak{Y}$ be subgroup closed group classes consisting of polycyclic-byfinite groups and such that $\mathfrak{X} \cap \mathfrak{Y} \leq \mathfrak{F}$. If $\mathfrak{X}$ and $\mathfrak{Y}$ are accessible, then also $\mathbf{L} \mathfrak{X} \cup \mathbf{L} \mathfrak{Y}$ is an accessible class.

Corollary 2.9 Let $\mathfrak{X}$ and $\mathfrak{Y}$ be subgroup closed group classes consisting of polycyclic-byfinite groups and such that $\mathfrak{X} \cap \mathfrak{Y} \leq \mathfrak{F}$. If all infinite locally graded opponents of $\mathfrak{X}$ and of $\mathfrak{Y}$ are not finitely generated, then the class $\mathbf{L} \mathfrak{X} \cup \mathbf{L} \mathfrak{Y}$ is accessible.

Corollary 2.10 Let $\mathfrak{X}$ be a subgroup closed group class consisting of polycyclic-by-finite groups. If all infinite locally graded opponents of $\mathfrak{X}$ are not finitely generated, then the class $\mathbf{L} \mathfrak{X} \cup \mathbf{L} \mathfrak{F}$ is accessible.

A combination of Theorem 2.4 and Corollary 2.10 provides the following interesting result which shows in particular that the classes $\mathbf{L} \mathfrak{N} \cup \mathbf{L} \mathfrak{F}$ and $\mathbf{L}\left(\mathbf{P}_{n} \mathscr{C}\right) \cup \mathbf{L} \mathfrak{F}$ are accessible.

Corollary 2.11 Let $\mathfrak{X}$ be a $\mathbf{P}_{n} \mathscr{C}$-Fitting group class which is subgroup closed. Then the class $\mathbf{L} \mathfrak{X} \cup \mathbf{L} \mathfrak{F}$ is accessible.

Notice also that $\mathfrak{N}_{c}=\mathbf{L}\left(\mathfrak{N}_{c} \cap\right.$ Max $)$ for any non-negative integer $c$ and hence the following result is a direct application of Corollary 2.10.

Corollary 2.12 For each non-negative integer c, the group class $\mathfrak{N}_{c} \cup \mathbf{L} \mathfrak{F}$ is accessible.

In contrast to the above result, the consideration of the locally dihedral 2-group shows that the class $\mathfrak{A} \cup \mathfrak{F}$ is inaccessible. On the other hand, there exist relevant proper subclasses $\mathfrak{X}$ of $\mathbf{L} \mathfrak{F}$ such that $\mathfrak{N}_{c} \cup \mathfrak{X}$ is accessible, like, for instance, the class $\mathfrak{E}$ of all Černikov groups.

Theorem 2.13 The group class $\mathfrak{N}_{c} \cup \mathfrak{C}$ is accessible for each non-negative integer $c$.

Proof Let $G$ be an infinite locally graded group whose proper subgroups belong to $\mathfrak{N}_{c} \cup \mathfrak{C}$. Then every finitely generated subgroup of $G$ contains a nilpotent subgroup of finite index and in particular $G$ is locally (nilpotent-by-finite). Moreover, it is clear that $G$ satisfies the minimal condition on subgroups that are not nilpotent of class at most $c$. As $\mathbf{L} \mathfrak{N}_{c}=\mathfrak{N}_{c}$, it follows that either $G$ is a Černikov group or it is nilpotent of class at most $c$ (see [27], Theorem $\mathrm{B}(\mathrm{ix})$ ). Therefore the class $\mathfrak{N}_{c} \cup \mathfrak{C}$ is accessible. 
Of course, the statements of Corollary 2.12 and Theorem 2.13 for $c=0$ give that the classes $\mathbf{L} \mathfrak{F}$ and $\mathfrak{E}$ are accessible.

Lemma 2.14 Let $\mathscr{K}$ be an infinite locally finite field. Then each of the groups $\operatorname{PSL}(2, \mathscr{K})$ and $S z(\mathscr{K})$ contains a proper subgroup of infinite rank which is not locally supersoluble.

Proof Let $p$ be the characteristic of the field $\mathscr{K}$. It is known that both groups $\operatorname{PSL}(2, \mathscr{K})$ and $\operatorname{Sz}(\mathscr{K})$ contain a Frobenius subgroup $X=C \ltimes N$, where the Frobenius kernel $N$ is isomorphic to the additive group of $\mathscr{K}$ and the Frobenius complement $C$ is a homomorphic image of the multiplicative group of $\mathscr{K}$ involving infinitely many primes (see, for instance, [26]). Clearly, $X$ has infinite rank, and it cannot be locally supersoluble since any element of $C$ of prime order $q>p$ does not centralize $N$.

Theorem 2.15 Let $\mathfrak{X}$ be a $\mathbf{P}_{n} \mathscr{C}$-Fitting class which is subgroup closed. Then the group class $\mathbf{L} \mathfrak{X} \cup \mathfrak{C}$ is accessible.

Proof Assume for a contradiction that $\mathbf{L} \mathfrak{X} \cup \mathfrak{C}$ admits an infinite locally graded opponent $G$. Then $G$ is locally finite by Corollary 2.11 and in particular it contains a finite subgroup $E$ which is not in $\mathfrak{X}$. Since every proper subgroup of $G$ is either locally supersoluble or Černikov, it follows easily from Lemma 2.14 and Theorem of [23] that $G$ has no infinite simple homomorphic images. In particular, either the centre $\zeta(G)$ has finite index or $G$ contains a normal subgroup $N$ such that $\zeta(G)<N<G$; if $N$ is Černikov, then $G / C_{G}(N)$ is likewise a Černikov group (see [28] Part 1, Theorem 3.29) and hence $C_{G}(N)$ is a proper normal subgroup of $G$ which is not Černikov. Thus $G$ contains in any case a non-Černikov normal subgroup $M$. It follows that $G=E M$, so $G / M$ is a finite group whose proper subgroups are supersoluble and hence it is soluble. Thus $G$ is locally soluble and so it contains an abelian subgroup $A=A_{1} \times A_{2}$, where both $A_{1}$ and $A_{2}$ are non-Černikov and $E \leq N_{G}\left(A_{1}\right) \cap N_{G}\left(A_{2}\right)$ (see [20]). Clearly, $E A_{1}$ and $E A_{2}$ are proper subgroups of $G$ which neither are Cernikov nor belong to $\mathbf{L} \mathfrak{X}$ and this contradiction completes the proof.

Corollary 2.16 The group classes $\mathbf{L} \mathfrak{N} \cup \mathfrak{C}$ and $\mathbf{L}\left(\mathbf{P}_{n} \mathscr{C}\right) \cup \mathfrak{C}$ are accessible.

For our purposes, we need the following result that was proved in [8] (see Proposition 1 and Theorem 2).

Lemma 2.17 Let $G$ be a countable simple locally (soluble-by-finite) group which is not locally finite. Then $G$ contains a locally soluble subgroup $R$ such that the Hirsch-Plotkin radical of $R$ is trivial and for each element $g$ of $G$ there exists a positive integer $n=n(g)$ such that $g^{n} \in R$. Moreover, $G$ is the union of a chain of proper subgroups containing $R$.

Theorem 2.18 The group class $\mathfrak{P} \mathfrak{\mathbf { L }} \cup \mathbf{L}$ is accessible.

Proof Assume for a contradiction that the statement is false and let $G$ be a locally graded opponent of $\mathfrak{P} \mathfrak{F} \cup \mathbf{L} \mathfrak{F}$. Clearly, $G$ cannot have proper subgroups of finite index, so that in particular it is not finitely generated. Since $G$ is not locally finite, it contains a finitely generated infinite subgroup $X$; moreover, there exists a countable subgroup $Y$ of $G$ which is not polycyclic-by-finite, since the class $\mathfrak{P} \mathfrak{F}$ is countably recognizable (see, for instance, 
[14]). Then $G=\langle X, Y\rangle$ is countable and obviously non-periodic. As $G$ is locally (solubleby-finite), it follows from Lemma 2.17 that $G$ is not simple.

If $G$ contains a maximal normal subgroup $M$, the factor group $G / M$ is simple, so that it cannot be an opponent of $\mathfrak{P} \mathfrak{F} \cup \mathbf{L} \mathfrak{F}$ and hence is locally finite. Then $M$ is not locally finite, so that it is polycyclic-by-finite and thus there exists a characteristic subgroup $K$ of $M$ such that $M / K$ is finite and $K / K^{\prime}$ is infinite. The factor group $G / C_{G}\left(K / K^{\prime}\right)$ is periodic, so even finite (see, for instance, [28] Part 1, p.85) and hence $C_{G}\left(K / K^{\prime}\right)=G$. It follows that $G / K^{\prime}$ is locally finite over its centre, whence $G^{\prime} / K^{\prime}$ is locally finite by the famous Schur's theorem (see [28] Part 1, Corollary to Theorem 4.12). On the other hand, $G / G^{\prime}$ is divisible and cannot contain subgroups isomorphic to the additive group of rational numbers, so that $G / G^{\prime}$ is periodic and hence $G / K^{\prime}$ is likewise periodic, which is a contradiction since $K / K^{\prime}$ is infinite. Therefore $G$ has no maximal normal subgroups.

An application of Zorn's lemma yields that there exists a chain

$$
L_{1}<L_{2}<\ldots<L_{n}<L_{n+1}<\ldots
$$

of proper normal subgroups of $G$ such that

$$
G=\bigcup_{n \in \mathbb{N}} L_{n} .
$$

Since $G$ is not locally finite, there is a positive integer $m$ such that $L_{m}$ is not locally finite. Then $L_{n}$ is polycyclic-by-finite for all $n \geq m$ and hence even for all $n$. On the other hand, the automorphism group of any polycyclic-by-finite group is residually finite (see, for instance, [28] Part 2, Corollary to Theorem 9.12) and then $L_{n} \leq \zeta(G)$ for all $n$. Therefore $G$ is abelian and so periodic, because it is divisible and cannot contain copies of the additive group of rational numbers. This last contradiction completes the proof.

\section{Corollary 2.19 The group class $\mathfrak{P} \mathfrak{F} \mathfrak{\mathfrak { C }}$ is accessible.}

Proof Let $G$ be any locally graded group whose proper subgroups belong to $\mathfrak{P} \mathfrak{F} \cup \mathfrak{C}$ and assume that $G$ is not polycyclic-by-finite. Then it follows from Theorem 2.18 that $G$ is locally finite and hence all proper subgroups of $G$ are Černikov. Therefore $G$ itself is a Černikov group because the class $\mathfrak{C}$ is accessible.

Notice that the above statement implies that Prüfer groups are the infinite locally graded opponents of $\mathfrak{P} \mathfrak{F}$.

Our next corollary shows in particular that in the statements of Theorem 2.18 and Corollary 2.19 the group class $\mathfrak{P} \mathfrak{F}$ can be replaced either by the class of supersolubleby-finite groups or by the class of finitely generated nilpotent-by-finite groups.

Corollary 2.20 Let $\mathfrak{X}$ be a subgroup closed group class consisting of polycyclic groups. Then the group classes $\mathfrak{X} \mathfrak{F} \cup \mathbf{L} \mathfrak{F}$ and $\mathfrak{X} \mathfrak{F} \cup \mathfrak{C}$ are accessible.

Proof Assume for a contradiction that there exists an infinite locally graded opponent $G$ of the group class $\mathfrak{X} \mathfrak{F} \cup \mathbf{L} \mathfrak{F}$ (the group class $\mathfrak{X} \mathfrak{F} \cup \mathfrak{C}$, respectively). Then $G$ is polycyclic-byfinite by Theorem 2.18 (Corollary 2.19, respectively) and so it contains a proper subgroup $X$ of finite index, which of course belongs to $\mathfrak{X} \mathfrak{F}$. Since $\mathfrak{X}$ is subgroup closed, it follows that $G$ is an $\mathfrak{X} \mathfrak{F}$-group, a contradiction. 
The following result applies in particular to the class $\mathfrak{P}$ and, by Theorem 2.4 , to any subgroup closed $\mathbf{P}_{n} \mathscr{C}$-Fitting group class.

Corollary 2.21 Let $\mathfrak{X}$ be a subgroup closed group class consisting of polycyclic groups and such that all its infinite locally graded opponents are not finitely generated. Then the group classes $\mathfrak{X} \cup \mathbf{L} \mathfrak{F}$ and $\mathfrak{X} \cup \mathfrak{C}$ are accessible.

Proof Assume for a contradiction that $G$ is an infinite locally graded opponent of one of the classes $\mathfrak{X} \cup \mathbf{L} \mathfrak{F}$ and $\mathfrak{X} \cup \mathfrak{C}$. Then $G$ is polycyclic-by-finite by Corollary 2.20 and so all its proper infinite subgroups belong to $\mathfrak{X}$. On the other hand, each finite subgroup of $G$ is contained in a proper subgroup of finite index and hence it is in $\mathfrak{X}$. Therefore $G$ is also an opponent of $\mathfrak{X}$, which is impossible because it is finitely generated.

Notice that, if $\mathfrak{X}$ is any group class, the accessibility of $\mathfrak{X} \cup \mathfrak{C}$ is equivalent to that of the class $\mathfrak{X} \cup \operatorname{Min}$ (to this aim, it is enough to observe that every locally graded group satisfying the minimal condition is locally finite and so even Černikov by a famous theorem of Sunkov [34]). In particular, the obvious fact that the class Min is accessible can be also obtained as a special case of the above results. More generally, if $\theta$ is any property pertaining to subgroups such that $X$ is a $\theta$-subgroup of $H$, whenever $X$ is a $\theta$-subgroup of a group $G$ and $X \leq H \leq G$, it turns out that the class of all groups satisfying the minimal condition on $\theta$-subgroups is accessible. In particular, if $\mathfrak{X}$ is any group class, the class of all groups satisfying the minimal condition on $\mathfrak{X}$-subgroups is accessible. Moreover, the class of all groups with the minimal condition on normal subgroups is accessible.

\section{Theorem 2.22 The group classes $\mathbf{L} \mathfrak{S}$ and $\mathbf{L} \mathfrak{S} \cup \mathbf{L} \mathfrak{F}$ are accessible.}

Proof Let $G$ be an infinite finitely generated locally graded group whose proper subgroups belong to $\mathbf{L} \mathfrak{S} \cup \mathbf{L} \mathfrak{F}$. Then $G$ contains a proper normal subgroup $N$ of finite index, which is finitely generated and hence soluble. Thus $G$ is soluble-by-finite.

Let $S$ be a soluble normal subgroup of finite index of $G$ of smallest derived length, so that in particular $S / S^{\prime}$ is infinite. If $q$ is a prime which does not divide $|G / S|$, it follows from the theorem of Schur-Zassenhaus applied to the finite group $G / S^{\prime} S^{q}$ that $G=E S$ and $E \cap S=S^{\prime} S^{q}$ for a suitable subgroup $E$. Then $E$ is a proper subgroup of finite index of $G$, so that $E$ is soluble and hence $G$ itself is soluble.

Since the classes $\mathbf{L} \mathfrak{S}$ and $\mathbf{L} \mathfrak{S} \cup \mathbf{L} \mathfrak{F}$ are local, all their opponents would be finitely generated, which is impossible by the first part of the proof. Therefore $\mathbf{L} \mathfrak{S}$ and $\mathbf{L} \mathfrak{S} \cup \mathbf{L} \mathfrak{F}$ are accessible.

It follows in particular from Theorem 2.22 and Lemma 2.1 that the class of soluble Černikov groups and the class of periodic locally soluble groups are accessible.

In contrast to the case of $\mathbf{P}_{n} \mathscr{C}$-Fitting classes described in Theorem 2.15 , in the statement of Theorem 2.22 the class $\mathbf{L} \mathfrak{F}$ cannot be replaced by $\mathfrak{C}$. In fact, if $\mathscr{K}$ is any infinite locally finite field with no infinite proper subfields, it is known that all infinite proper subgroups of the infinite locally finite simple group $\operatorname{PSL}(2, \mathscr{K})$ are metabelian and hence $\operatorname{PSL}(2, \mathscr{K})$ is an opponent of $\mathfrak{X} \cup \mathfrak{C}$ for each group class $\mathfrak{X}$ such that $\mathfrak{A}^{2} \leq \mathfrak{X} \leq \mathbf{L} \mathfrak{S}$. The same example also shows that the class $\mathfrak{X} \cup \mathfrak{F}$ is inaccessible for the same choices of $\mathfrak{X}$; in particular, the class $\mathfrak{S} \cup \mathfrak{F}$ is inaccessible while, as we remarked in the introduction, it seems to be unknown whether soluble groups form an accessible class or not. 
The last part of this section deals with a couple of group classes of a different kind. Recall that a group $G$ is called metahamiltonian if all its non-abelian subgroups are normal, while $G$ is called quasihamiltonian if $X Y=Y X$ for all subgroups $X$ and $Y$ of $G$. Metahamiltonian groups were introduced and studied by Romalis and Sesekin (see [29-31]), who proved in particular that the commutator subgroup of any soluble metahamiltonian group is finite of prime-power order. Of course, all subgroups of a metahamiltonian group are likewise metahamiltonian, and it is known that the class $\mathscr{H}$ of metahamiltonian groups is local and accessible (see [5]). As concerns quasihamiltonian groups, it is well known that these groups are locally nilpotent and have a modular subgroup lattice (see, for instance, [32], Theorem 6.2.10); moreover, the class $\mathscr{Q}$ of all quasihamiltonian groups is local and so also accessible by Lemma 2.3 of [9].

It has been recently proved in [11] that if a locally graded group $G$ satisfies the minimal condition on subgroups which are not metahamiltonian (quasihamiltonian), then $G$ is either metahamiltonian (quasihamiltonian) or a Černikov group. Thus the group classes $\mathscr{H} \cup \mathfrak{C}$ and $\mathscr{Q} \cup \mathfrak{C}$ are accessible. Moreover, by choosing $\mathfrak{V}=\mathfrak{F}$ and either $\mathfrak{X}=\mathscr{H} \cap \operatorname{Max}$ or $\mathfrak{X}=\mathscr{Q} \cap \operatorname{Max}$ in the statement of Lemma 2.6, we get the following result.

Corollary 2.23 The group classes $\mathscr{H} \cup \mathbf{L} \mathfrak{F}$ and $\mathscr{Q} \cup \mathbf{L} \mathfrak{F}$ are accessible.

\section{The accessible closure}

This section deals with the embedding of an arbitrary group class into a smallest accessible group class; this is the real core of the paper.

Let $\mathfrak{X}$ be a class of groups and put $\mathbf{A}_{0}(\mathfrak{X})=\mathfrak{X}$. If $\alpha>0$ is an ordinal number such that the group class $A_{\beta}(\mathfrak{X})$ has been defined for each ordinal $\beta<\alpha$, denote by $\mathbf{A}_{\alpha}(\mathfrak{X})$ the class consisting of all groups in $\mathbf{A}_{\alpha-1}(\mathfrak{X})$ and of all infinite locally graded opponents of $\mathbf{A}_{\alpha-1}(\mathfrak{X})$ when $\alpha$ is successor, while write

$$
\mathbf{A}_{\alpha}(\mathfrak{X})=\bigcup_{\beta<\alpha} \mathbf{A}_{\beta}(\mathfrak{X})
$$

when $\alpha$ is limit. Of course, $\mathfrak{X} \leq \mathbf{A}_{\gamma}(\mathfrak{X}) \leq \mathbf{A}_{\delta}(\mathfrak{X})$ whenever $\gamma \leq \delta$. The accessible closure of $\mathfrak{X}$ is now defined as

$$
\mathbf{A}(\mathfrak{X})=\bigcup_{\alpha} \mathbf{A}_{\alpha}(\mathfrak{X})
$$

where $\alpha$ ranges over all ordinal numbers.

It follows from the above construction that $\mathbf{A}(\mathfrak{X}) \backslash \mathfrak{X}$ consists only of infinite locally graded groups; in particular, if all groups in $\mathfrak{X}$ are locally graded, then $\mathbf{A}(\mathfrak{X})$ is contained in the class of locally graded groups. Notice also that every infinite locally graded opponent of $\mathfrak{X}$ belongs to $\mathbf{A}(\mathfrak{X})$.

The following result validates the name accessible closure.

Theorem 3.1 Let $\mathfrak{X}$ be a group class. Then $\mathbf{A}(\mathfrak{X})$ is accessible and $\mathbf{A}(\mathfrak{X}) \leq \mathfrak{V}$ for every accessible group class $\mathfrak{Y}$ containing $\mathfrak{X}$. 
Proof Assume for a contradiction that $G$ is an infinite locally graded opponent of $\mathbf{A}(\mathfrak{X})$. Since $G$ is a set, there exists an ordinal $\lambda$ such that all proper subgroups of $G$ lie in $\mathbf{A}_{\lambda}(\mathfrak{X})$, so that $G$ is also an opponent of $\mathbf{A}_{\lambda}(\mathfrak{X})$ and hence it belongs to $\mathbf{A}_{\lambda+1}(\mathfrak{X}) \leq \mathbf{A}(\mathfrak{X})$, which is impossible. Therefore $\mathbf{A}(\mathfrak{X})$ is an accessible group class.

Let $\mathfrak{Y}$ be any accessible group class for which $\mathfrak{X} \leq \mathfrak{V}$ and assume for a contradiction that $\mathbf{A}(\mathfrak{X})$ is not contained in $\mathfrak{Y}$. Consider the least ordinal $\mu$ such that $\mathbf{A}_{\mu}(\mathfrak{X})$ is not a subclass of $\mathfrak{Y}$ and let $H$ be a group in $\mathbf{A}_{\mu}(\mathfrak{X}) \backslash \mathfrak{Y}$. Clearly, $\mu>0$ is not a limit ordinal and $\mathbf{A}_{\mu-1}(\mathfrak{X}) \leq \mathfrak{V}$, so that, by the definition of $\mathbf{A}_{\mu}(\mathfrak{X})$, it turns out that $H$ is an infinite locally graded opponent of $\mathbf{A}_{\mu-1}(\mathfrak{X})$. Thus $H$ is also an opponent of $\mathfrak{Y}$, which is impossible as $\mathfrak{Y}$ ) is accessible.

Corollary 3.2 A group class $\mathfrak{X}$ is accessible if and only if $\mathbf{A}(\mathfrak{X})=\mathfrak{X}$.

Corollary 3.3 Let $\mathfrak{X}$ and $\mathfrak{Y}$ be group classes such that $\mathfrak{X} \leq \mathfrak{V}$. Then $\mathbf{A}(\mathfrak{X}) \leq \mathbf{A}(\mathfrak{Y})$.

Proof As the class $\mathbf{A}(\mathfrak{Y})$ is accessible by Theorem 3.1 and $\mathfrak{X} \leq \mathfrak{Y} \leq \mathbf{A}(\mathfrak{Y})$ ), it follows again from Theorem 3.1 that $\mathbf{A}(\mathfrak{X}) \leq \mathbf{A}(\mathfrak{Y})$ ).

Notice that if $\mathfrak{X}$ is any inaccessible group class, then $\mathfrak{X}<\mathbf{A}(\mathfrak{X})$ but obviously we have $\mathbf{A}(\mathfrak{X})=\mathbf{A}(\mathbf{A}(\mathfrak{X}))$ and hence in the above statement $\leq$ cannot be replaced by $<$.

Our next purpose is to prove that there exists a local group class $\mathfrak{X}$ which is subgroup closed and whose accessible closure $\mathbf{A}(\mathfrak{X})$ is not local. To see this, consider a cohopfian finitely generated torsion-free nilpotent group $W$ (see Sect. 2) and put

$$
G=W \times \underset{p}{\operatorname{Dr}} C_{p}
$$

where $C_{p}$ is a group of order $p$ and $p$ ranges over all prime numbers. Let $\mathfrak{X}$ be the class consisting of all groups whose finitely generated subgroups are isomorphic to subgroups of $G$ not containing $W$. Clearly, $\mathfrak{X}$ is a subgroup closed local class and $W$ belongs to $\mathbf{A}_{1}(\mathfrak{X}) \backslash \mathfrak{X}$. More generally, it can be easily proved by induction that, for each positive integer $n$ and for any choice $p_{1}, \ldots, p_{n}$ of $n$ prime numbers, the group

$$
W \times C_{p_{1}} \times \ldots \times C_{p_{n}}
$$

is a member of the class $\mathbf{A}_{n+1}(\mathfrak{X}) \backslash \mathbf{A}_{n}(\mathfrak{X})$. It follows that, for any non-negative integer $n$, the group $G$ cannot be an opponent of $\mathbf{A}_{n}(\mathfrak{X})$, so it is not in $\mathbf{A}_{n+1}(\mathfrak{X})$ and hence neither in $\mathbf{A}_{\omega}(\mathfrak{X})$. On the other hand, all finitely generated subgroups of $G$ belong to $\mathbf{A}_{\omega}(\mathfrak{X})$ and so $\mathbf{L}\left(\mathbf{A}_{\omega}(\mathfrak{X})\right) \neq \mathbf{A}_{\omega}(\mathfrak{X})$. Assume for a contradiction that there exists an infinite set $\pi$ of primes such that the group

$$
W \times\left(\operatorname{Dr}_{p \in \pi} C_{p}\right)
$$

belongs to $\mathbf{A}(\mathfrak{X})$, and let $\mu$ be the least ordinal number for which $\mathbf{A}_{\mu}(\mathfrak{X})$ contains a group $H$ of this type. Clearly, $\mu>0$ and $\mu$ is not a limit, so that $H$ must be an opponent of $\mathbf{A}_{\mu-1}(\mathfrak{X})$, which is of course impossible since any maximal subgroup of $H$ containing $W$ does not belong to $\mathbf{A}_{\mu-1}(\mathfrak{X})$. In particular, $G$ does not belong to $\mathbf{A}(\mathfrak{X})$, while all its finitely generated subgroups lie in $\mathbf{A}_{\omega}(\mathfrak{X}) \leq \mathbf{A}(\mathfrak{X})$. Therefore the class $\mathbf{A}(\mathfrak{X})$ is not local. Although this argument proves that $\mathbf{A}_{\alpha}(\mathfrak{X})$ is not local for each infinite ordinal $\alpha$, our next statement shows that the situation is better for finite ordinal numbers. 
Proposition 3.4 If $\mathfrak{X}$ is a local group class, then also the class $\mathbf{A}_{n}(\mathfrak{X})$ is local for each nonnegative integer $n$.

Proof Assume for a contradiction that the statement is false and let $m$ be the smallest nonnegative integer such that the class $\mathbf{A}_{m}(\mathfrak{X})$ is not local. Clearly, $m>0$ and $\mathbf{A}_{m-1}(\mathfrak{X})$ is local. Let $G$ be a group in $\mathbf{L}\left(\mathbf{A}_{m}(\mathfrak{X})\right) \backslash \mathbf{A}_{m}(\mathfrak{X})$ and notice that $G$ cannot be finitely generated. Moreover, since $\mathbf{A}_{m-1}(\mathfrak{X})$ is local and $G$ is not in $\mathbf{A}_{m-1}(\mathfrak{X})$, there exists a finitely generated subgroup $E$ of $G$ which is not contained in any $\mathbf{A}_{m-1}(\mathfrak{X})$-subgroup of $G$. If $g$ is an element of $G \backslash E$, the subgroup $\langle g, E\rangle$ lies in a subgroup $U$ of $G$ that belongs to $\mathbf{A}_{m}(\mathfrak{X})$. Obviously, $U$ cannot belong to $\mathbf{A}_{m-1}(\mathfrak{X})$, so that it is an opponent of $\mathbf{A}_{m-1}(\mathfrak{X})$ and in particular its proper subgroup $E$ is in $\mathbf{A}_{m-1}(\mathfrak{X})$. This contradiction completes the proof of the statement.

In contrast to the behaviour of locality, it can be proved that the accessible closure of any subgroup closed group class is likewise subgroup closed.

Proposition 3.5 If $\mathfrak{X}$ is a subgroup closed group class, then also the class $\mathbf{A}_{\alpha}(\mathfrak{X})$ is subgroup closed for each ordinal $\alpha$. In particular, the accessible closure of $\mathfrak{X}$ is subgroup closed.

Proof Assume for a contradiction that the statement is false and let $\mu$ be the smallest ordinal such that $\mathbf{A}_{\mu}(\mathfrak{X})$ is not subgroup closed. Consider a group $X$ which is not in $\mathbf{A}_{\mu}(\mathfrak{X})$ but is isomorphic to a (proper) subgroup of some group $G \in \mathbf{A}_{\mu}(\mathfrak{X})$. Clearly, $\mu>0$ is not a limit and the class $\mathbf{A}_{\mu-1}(\mathfrak{X})$ is subgroup closed, so that $G$ cannot belong to $\mathbf{A}_{\mu-1}(\mathfrak{X})$. Therefore $G$ is an opponent of $\mathbf{A}_{\mu-1}(\mathfrak{X})$, and hence $X \in \mathbf{A}_{\mu-1}(\mathfrak{X}) \leq \mathbf{A}_{\mu}(\mathfrak{X})$, a contradiction.

Any group class $\mathfrak{X}$ naturally lies between two subgroup closed classes of groups. In fact, we may consider the class $\mathbf{S} \mathfrak{X}$ consisting of all groups which are embeddable in an $\mathfrak{X}$-group and the class $\mathfrak{X}^{\mathrm{S}}$ of all groups whose subgroups belong to $\mathfrak{X}$. Then $\mathbf{S} \mathfrak{X}$ is the smallest subgroup closed group class containing $\mathfrak{X}$ and $\mathfrak{X}^{\mathrm{S}}$ is the largest subgroup closed group class contained in $\mathfrak{X}$; in particular, $\mathfrak{X}^{\mathrm{S}}=\mathfrak{X}=\mathbf{S} \mathfrak{X}$ if and only if the group class $\mathfrak{X}$ is subgroup closed. The relation between these operators and the operator $\mathfrak{X} \mapsto \mathbf{A}(\mathfrak{X})$ is described by the following consequence of Proposition 3.5.

Corollary 3.6 Let $\mathfrak{X}$ be a group class. Then

$$
\mathbf{A}\left(\mathfrak{X}^{\mathrm{S}}\right) \leq(\mathbf{A}(\mathfrak{X}))^{\mathrm{S}} \leq \mathbf{A}(\mathfrak{X}) \leq \mathbf{S}(\mathbf{A}(\mathfrak{X})) \leq \mathbf{A}(\mathbf{S}(\mathfrak{X})) .
$$

Proof Since $\mathfrak{X}^{\mathrm{S}} \leq \mathfrak{X} \leq \mathbf{S} \mathfrak{X}$, we have

$$
\mathbf{A}\left(\mathfrak{X}^{\mathrm{S}}\right) \leq \mathbf{A}(\mathfrak{X}) \leq \mathbf{A}(\mathbf{S} \mathfrak{X})
$$

by Corollary 3.3. Moreover, the group classes $\mathbf{A}\left(\mathfrak{X}^{\mathrm{S}}\right)$ and $\mathbf{A}(\mathbf{S} \mathfrak{X})$ are subgroup closed by Proposition 3.5, and so the statement follows.

It is not difficult to see that the group classes $\mathfrak{X}$ and $\mathfrak{X}^{\mathrm{S}}$ have the same opponents, so $\mathfrak{X}$ is accessible if and only if $\mathfrak{X}^{\mathrm{S}}$ is accessible. In contrast to this fact, there exists a group class $\mathfrak{Y}$ such that $\mathbf{A}\left(\mathfrak{Y}^{\mathrm{S}}\right) \neq \mathbf{A}(\mathfrak{Y})^{\mathrm{S}}$. To see this, let $\mathfrak{X}$ be the subgroup closed local 
group class constructed after Corollary 3.3 such that $\mathbf{A}(\mathfrak{X})$ is not local and let $\mathfrak{Y}$ be the class obtained by adding to $\mathfrak{X}$ all groups which are isomorphic to groups of the form

$$
W \times\left(\operatorname{Dr}_{p \in \pi} C_{p}\right),
$$

where $\pi$ is any infinite set of primes. Then $\mathfrak{Y}^{\mathrm{S}}=\mathfrak{X}$ and so $G$ does not belong to $\mathbf{A}\left(\mathfrak{V}^{\mathrm{S}}\right)$. On the other hand, every subgroup of $G$ which is not finitely generated belongs to $\mathfrak{Y}$ ) and hence all subgroups of $G$ lie in $\mathbf{A}_{\omega}(\mathfrak{Y})$ ). It follows that $G$ is a member of the largest subgroup closed subclass $(\mathbf{A}(\mathfrak{Y}))^{\mathrm{S}}$ of $\mathbf{A}(\mathfrak{Y})$, and hence $\mathbf{A}\left(\mathfrak{Y}^{\mathrm{S}}\right) \neq(\mathbf{A}(\mathfrak{Y}))^{\mathrm{S}}$.

Notice now that the group classes $\mathfrak{X}$ and $\mathbf{S} \mathfrak{X}$ need not to have the same (infinite locally graded) opponents. In fact, let $p$ and $q$ be distinct prime numbers and let $\mathfrak{X}$ be the class consisting of the trivial groups, of all Prüfer $p$-groups and of all finite cyclic groups of order $p^{n} q$ for some non-negative integer $n$. Then $\mathfrak{X}$ is accessible, while $C_{p^{\infty}} \times C_{q}$ is an opponent of $\mathbf{S} \mathfrak{X}$ and $\mathbf{S}(\mathbf{A}(\mathfrak{X}))=\mathbf{S} \mathfrak{X} \neq \mathbf{A}(\mathbf{S} \mathfrak{X})$.

The above two examples show that all inequalities in the statement of Corollary 3.6 may be strict.

As we remarked in Sect. 2 the union of two accessible and subgroup closed group classes $\mathfrak{X}$ and $\mathfrak{Y}$ need not be accessible, so that in general $\mathbf{A}(\mathfrak{X}) \cup \mathbf{A}(\mathfrak{V})$ is properly contained in $\mathbf{A}(\mathfrak{X} \cup \mathfrak{Y})$ ). On the other hand, the operator $\mathbf{A}$ behaves better on intersections and differences.

Lemma 3.7 If $\mathfrak{X}, \mathfrak{Y}$ are subgroup closed group classes, then $\mathbf{A}(\mathfrak{X} \cap \mathfrak{Y})=\mathbf{A}(\mathfrak{X}) \cap \mathbf{A}(\mathfrak{Y})$ ).

Proof Since the intersection $\mathbf{A}(\mathfrak{X}) \cap \mathbf{A}(\mathfrak{Y})$ is an accessible class containing $\mathfrak{X} \cap \mathfrak{Y})$, we have $\mathbf{A}(\mathfrak{X} \cap \mathfrak{Y}) \leq \mathbf{A}(\mathfrak{X}) \cap \mathbf{A}(\mathfrak{Y})$. Assume for a contradiction that the inclusion is strict and let $\mu$ be the least ordinal number such that $\mathbf{A}_{\mu}(\mathfrak{X}) \cap \mathbf{A}(\mathfrak{Y})$ contains a group $G$ which is not in $\mathbf{A}(\mathfrak{X} \cap \mathfrak{Y})$. Of course, $\mu$ is not limit. If $\mu>0$, then all proper subgroups of $G$ belong to $\mathbf{A}_{\mu-1}(\mathfrak{X})$, so also to $\mathbf{A}_{\mu-1}(\mathfrak{X}) \cap \mathbf{A}(\mathfrak{Y}) \leq \mathbf{A}(\mathfrak{X} \cap \mathfrak{Y})$. This is impossible because $\mathbf{A}(\mathfrak{X} \cap \mathfrak{Y})$ is accessible. Therefore $\mu=0$ and we may consider the least ordinal $\nu$ such that $\mathfrak{X} \cap \mathbf{A}_{v}(\mathfrak{Y})$ contains a group $H$ which is not in $\mathbf{A}(\mathfrak{X} \cap \mathfrak{Y})$. Now, $v>0$ and it is not limit, so that all proper subgroups of $H$ lie in $\mathfrak{X} \cap \mathbf{A}_{v-1}(\mathfrak{Y}) \leq \mathbf{A}(\mathfrak{X} \cap \mathfrak{Y})$ and hence $H$ itself belongs to $\mathbf{A}(\mathfrak{X} \cap \mathfrak{Y})$, the final contradiction.

In the above result the condition that both group classes $\mathfrak{X}$ and $\mathfrak{Y}$ ) are subgroup closed cannot be weakened. To see this, fix a prime number $p$ and choose as $\mathfrak{Y}$ ) the class consisting of all identity groups and all Prüfer $p$-groups; if $\mathfrak{X}$ is the class of all cyclic $p$-groups, then $\mathbf{A}(\mathfrak{X} \cap \mathfrak{Y})$ consists only of identity groups while

$$
\mathbf{A}(\mathfrak{X}) \cap \mathbf{A}(\mathfrak{Y})=\mathbf{A}(\mathfrak{X}) \cap \mathfrak{Y}=\mathfrak{Y} .
$$

On the other hand, the argument of the proof of Lemma 3.7 can be employed to prove the following result.

Lemma 3.8 Let $\mathfrak{Y}$ be a class of groups which is accessible and subgroup closed. Then $\mathbf{A}(\mathfrak{X} \cap \mathfrak{Y})=\mathbf{A}(\mathfrak{X}) \cap \mathfrak{Y}$ for each group class $\mathfrak{X}$.

Lemma 3.9 Let $\mathfrak{X}$ be a group class. Then $\mathbf{A}(\mathfrak{X} \backslash \mathfrak{Y}) \leq \mathbf{A}(\mathfrak{X}) \backslash \mathfrak{V}$ for every subgroup closed group class $\mathfrak{Y}$. 
Proof Assume for a contradiction that the statement is false and let $\mu$ be the least ordinal such that $\mathbf{A}_{\mu}(\mathfrak{X} \backslash \mathfrak{Y})$ contains a group $G$ which is not in $\mathbf{A}(\mathfrak{X}) \backslash \mathfrak{Y}$. Of course, $\mu>0$ is not limit. Then all proper subgroups of $G$ lie in $\mathbf{A}_{\mu-1}(\mathfrak{X} \backslash \mathfrak{Y}) \leq \mathbf{A}(\mathfrak{X}) \backslash \mathfrak{Y}$, so $G$ itself cannot belong to the subgroup closed class $\mathfrak{Y}$. Since $\mathbf{A}(\mathfrak{X})$ is accessible, $G$ belongs to $\mathbf{A}(\mathfrak{X})$ and so to $\mathbf{A}(\mathfrak{X}) \backslash \mathfrak{Y}$. This contradiction proves the statement.

Notice that the inclusion in the above result can be strict. To see this, let $\mathfrak{X}$ be the class consisting of all cyclic $p$-groups, where $p$ is a prime number, and let $\mathfrak{V}$ be the class of all cyclic $p$-groups of order at most $p^{k}$ for a fixed positive integer $k$; then the Prüfer $p$-group belongs to $\mathbf{A}(\mathfrak{X}) \backslash \mathfrak{Y}$ but not to $\mathbf{A}(\mathfrak{X} \backslash \mathfrak{V})$.

Corollary 3.10 Let $\mathfrak{X}$ be an accessible group class. Then $\mathfrak{X} \backslash \mathfrak{Y}$ is accessible for every subgroup closed group class $\mathfrak{Y}$.

Proof By Lemma 3.9 we have

$$
\mathfrak{X} \backslash \mathfrak{V} \leq \mathbf{A}(\mathfrak{X} \backslash \mathfrak{V}) \leq \mathbf{A}(\mathfrak{X}) \backslash \mathfrak{Y}=\mathfrak{X} \backslash \mathfrak{Y}
$$

and hence $\mathfrak{X} \backslash \mathfrak{Y}=\mathbf{A}(\mathfrak{X} \backslash \mathfrak{Y})$ is accessible.

Remark that the above statement is false for an arbitrary group class $\mathfrak{Y}$. In fact, for any prime number $p$, the class $\mathfrak{X}$ of all locally cyclic $p$-groups is accessible, while $\mathfrak{X} \backslash \mathfrak{V}$ is inaccessible if $\mathfrak{Y}$ is the class of all Prüfer $p$-groups.

Finally, there are group classes $\mathfrak{X}$ and $\mathfrak{Y}$ such that $\mathfrak{X}<\mathfrak{V}$ and $\mathbf{A}_{1}(\mathfrak{X}) \backslash \mathfrak{X}=\mathbf{A}_{1}(\mathfrak{Y}) \backslash \mathfrak{Y}$, but $\mathbf{A}(\mathfrak{X}) \backslash \mathfrak{X} \neq \mathbf{A}(\mathfrak{Y}) \backslash \mathfrak{V}$. In fact, as we already mentioned, it has been proved in [13] that the class $\mathbb{Z}$ of all hypercentral groups and the class $\dot{\mathbf{P}}_{n} \mathscr{C}$ of all hypercyclic groups have the same infinite locally graded opponents and all such opponents are periodic, in other words

$$
\mathbf{A}_{1}(\boldsymbol{Z}) \backslash \mathfrak{Z}=\mathbf{A}_{1}\left(\dot{\mathbf{P}}_{n} \mathscr{C}\right) \backslash \dot{\mathbf{P}}_{n} \mathscr{C}
$$

Consider now, for a prime number $p$, a $p$-group $H$ of Heineken-Mohamed type (see Sect. 2) and let $q_{1}, q_{2}$ be prime numbers different from $p$ and such that $q_{2}$ divides $q_{1}-1$. Put $K=H \times C_{q_{1}}$ and $G=\langle x\rangle \ltimes K$, where $x$ is an element of order $q_{2}$ acting trivially on $H$ and as a non-trivial automorphism on $C_{q_{1}}$. Then $G$ belongs to the class $\mathbf{A}_{3}\left(\dot{\mathbf{P}}_{n} \mathscr{C}\right) \leq \mathbf{A}\left(\dot{\mathbf{P}}_{n} \mathscr{C}\right)$. On the other hand, the class $\mathbf{A}(\boldsymbol{Z})$ is contained in $\mathbf{L} \mathfrak{N}$ by Theorem 3.1 and hence $G$ does not belong to $\mathbf{A}(\boldsymbol{Z})$. Moreover, $\mathbf{A}(\boldsymbol{Z}) \leq \mathbf{A}\left(\mathbf{P}_{n} \mathscr{C}\right)$ by Corollary 3.3 , and $\mathbf{A}(\boldsymbol{Z}) \backslash \mathbb{Z}$ cannot contain hypercyclic groups because every hypercyclic locally nilpotent group is hypercentral. Therefore $\mathbf{A}(\mathcal{Z}) \backslash \mathcal{Z}$ is a proper subclass of $\mathbf{A}\left(\hat{\mathbf{P}}_{n} \mathscr{C}\right) \backslash \mathbf{P}_{n} \mathscr{C}$.

\section{Examples of accessible closures}

This section is devoted to show the essential role played by Černikov groups in the determination of the accessible closure of some natural group classes containing all finite groups.

Recall that any Černikov group $G$ contains a divisible abelian normal subgroup $J(G)$, which is the direct product of a finite number $h=h(G)$ of Prüfer groups such that the factor group $G / J(G)$ is finite of order $k=k(G)$, say. The integers $h \geq 0$ and $k \geq 1$ are invariants of $G$, and the pair $s(G)=(h, k)$ is usually called the size of $G$. These pairs of integers can be ordered lexicographically by putting $(h, k) \leq\left(h^{\prime}, k^{\prime}\right)$ if and 
only if either $h<h^{\prime}$ or $h=h^{\prime}$ and $k \leq k^{\prime}$. Of course, $G$ is finite if and only if $h=0$, and $s(X)<s(G)$ whenever $X$ is a proper subgroup of an arbitrary group $G$.

The first result of this section proves that the accessible closure of the class $\mathfrak{F}$ of finite groups is not too large.

Theorem 4.1 The accessible closure of the class of finite groups is the class of Černikov groups.

Proof Since the class $\mathfrak{E}$ of Černikov groups is accessible, we have $\mathbf{A}(\mathfrak{F}) \leq \mathfrak{C}$ by Theorem 3.1. Assume for a contradiction that $\mathbf{A}(\mathfrak{F}) \neq \mathfrak{E}$ and let $G$ be a Černikov group of smallest possible size which is not in $\mathbf{A}(\mathfrak{F})$. Then all proper subgroups of $G$ belong to $\mathbf{A}(\mathfrak{F})$ and hence there exists an ordinal $\mu$ such that $\mathbf{A}_{\mu}(\mathfrak{F})$ contains all proper subgroups of $G$. Obviously, $G$ is an opponent of $\mathbf{A}_{\mu}(\mathfrak{F})$ and so it belongs to $\mathbf{A}_{\mu+1}(\mathfrak{F}) \leq \mathbf{A}(\mathfrak{F})$. This contradiction completes the proof.

The following result is a direct consequence of Theorem 4.1 and Lemma 3.8.

Corollary 4.2 If $\pi$ is any set of primes, the accessible closure of the class of finite $\pi$-groups is the class of Černikov $\pi$-groups.

We now describe the accessible closure of the most relevant classes consisting of polycyclic-by-finite groups.

Theorem 4.3 Let $\mathfrak{X}$ be a subgroup closed group class consisting of polycyclic groups. Then the accessible closure of $\mathfrak{X} \mathfrak{F}$ is the class $\mathfrak{X} \mathfrak{F} \cup \mathfrak{C}$.

Proof By Theorem 4.1 we have $\mathfrak{E}=\mathbf{A}(\mathfrak{F}) \leq \mathbf{A}(\mathfrak{X} \mathfrak{F})$. Then $\mathfrak{X} \mathfrak{F} \cup \mathfrak{S} \leq \mathbf{A}(\mathfrak{X} \mathfrak{F})$, and hence $\mathbf{A}(\mathfrak{X} \mathfrak{F})=\mathfrak{X} \mathfrak{F} \cup \mathfrak{C}$ because this latter class is accessible by Corollary 2.20.

The above result applies in particular to the group classes consisting of polycyclic groups, supersoluble groups, finitely generated nilpotent groups.

\section{Corollary 4.4 The following equalities hold:}

(a) $\mathbf{A}(\mathfrak{P} \mathfrak{F})=\mathfrak{P} \mathfrak{F} \cup \mathfrak{C}$;

(b) $\mathbf{A}\left(\left(\mathbf{P}_{n}^{\mathscr{C}}\right) \mathfrak{F}\right)=\left(\mathbf{P}_{n}^{\mathscr{C}}\right) \mathfrak{F} \cup \mathfrak{C}$

(c) $\mathbf{A}(\mathfrak{N} \mathfrak{F} \cap \operatorname{Max})=(\mathfrak{N} \mathfrak{F} \cap \operatorname{Max}) \cup \mathfrak{C}$.

It was already noticed that the class of locally soluble groups, the class of locally supersoluble groups and the class of locally nilpotent groups are accessible. Moreover, since $\mathfrak{P}=\mathfrak{P} \mathfrak{F} \cap \mathbf{L} \mathfrak{S}, \mathbf{P}_{n} \mathscr{C}=\left(\mathbf{P}_{n} \mathscr{C}\right) \mathfrak{F} \cap \mathbf{L}\left(\mathbf{P}_{n} \mathscr{C}\right)$ and $\mathfrak{N} \cap \operatorname{Max}=(\mathfrak{N} \mathfrak{F} \cap \operatorname{Max}) \cap \mathbf{L} \mathfrak{N}$, the following result is a direct consequence of Lemma 3.8 and Corollary 4.4. 


\section{Corollary 4.5 The following equalities hold:}

(a) $\mathbf{A}(\mathfrak{P})=\mathfrak{P} \cup(\mathfrak{C} \cap \mathfrak{S})$;

(b) $\mathbf{A}\left(\mathbf{P}_{n} \mathscr{C}\right)=\mathbf{P}_{n} \mathscr{C} \cup\left(\mathfrak{C} \cap \mathbf{L}\left(\mathbf{P}_{n} \mathscr{C}\right)\right)$;

(c) $\mathbf{A}(\mathfrak{N} \cap \operatorname{Max})=(\mathfrak{N} \cap \operatorname{Max}) \cup(\mathfrak{C} \cap \mathbf{L} \mathfrak{N})=\mathbf{L} \mathfrak{N} \cap(\mathfrak{P} \cup \mathfrak{C})$.

Recall that a group $G$ is said to be an $F C$-group if every element of $G$ has only finitely many conjugates, or equivalently if the centralizer $C_{G}(g)$ has finite index in $G$ for each element $g$ of $G$. Abelian groups and finite groups obviously have the $F C$-property and $F C$ groups were introduced with the aim of studying common properties of these two important group classes. We refer to the monograph [35] for a detailed account of this relevant part of the theory of infinite groups.

The consideration of the locally dihedral 2-group shows that $F C$-groups form an inaccessible class. On the other hand, it is known that every locally graded opponent of the class of $F C$-groups is either Černikov or a countable perfect locally finite $p$-group with no proper subgroups of finite index (see [24] and [35], Theorem 8.11). The existence of groups of the latter type seems to be still an open question; this is of course the main obstruction in the determination of the accessible closure of the class of $F C$-groups. Thus, in the following, we construct the accessible closures of certain special classes of groups with finite conjugacy classes. Our next result deals in particular, when $c=1$, with the class of groups with boundedly finite conjugacy classes (that is well known to coincide with the class $\mathfrak{F} \mathfrak{A}$ of groups with a finite commutator subgroup) and shows that $\mathbf{A}(\mathfrak{F} \mathfrak{A})=\mathfrak{F} \mathfrak{A} \cup \mathfrak{C}$.

Corollary 4.6 $\mathbf{A}\left(\mathfrak{F} \mathfrak{N}_{c}\right)=\mathfrak{F} \mathfrak{N}_{c} \cup \mathfrak{C}$ for each non-negative integer $c$.

Proof By Theorem 4.1 we have $\mathfrak{C}=\mathbf{A}(\mathfrak{F}) \leq \mathbf{A}\left(\mathfrak{F N}_{c}\right)$ and so $\mathfrak{F}_{c} \cup \mathfrak{C} \leq \mathbf{A}\left(\mathfrak{F} \mathfrak{N}_{c}\right)$. On the other hand, it follows from Theorem 1 of [4] that the class $\mathfrak{F} \mathfrak{N}_{c} \cup \mathfrak{C}$ is accessible and hence $\mathbf{A}\left(\mathfrak{F} \mathfrak{N}_{c}\right)=\mathfrak{F N}_{c} \cup \mathfrak{S}$

The class $\mathfrak{F} \mathfrak{U}$ is strictly related to the class $\mathbf{Z} \mathfrak{F}$ of all groups which are finite over the centre and actually Schur's theorem just states that $\mathbf{Z} \mathfrak{F}$ is contained in $\mathfrak{F} \mathfrak{A}$.

Corollary 4.7 The accessible closure $\mathbf{A}(\mathbf{Z} \mathfrak{F})$ of the class of all groups which are finite over the centre is the class $\mathbf{Z} \mathfrak{F} \cup \mathfrak{C}$.

Proof By Theorem 4.1 we have $\mathfrak{C}=\mathbf{A}(\mathfrak{F}) \leq \mathbf{A}(\mathbf{Z} \mathfrak{})$ and so $\mathbf{Z} \mathfrak{} \cup \mathfrak{C} \leq \mathbf{A}(\mathbf{Z} \mathfrak{})$. Assume for a contradiction that there exists a locally graded opponent $G$ of $\mathbf{Z} \mathfrak{F} \cup \mathfrak{C}$. Since $\mathbf{Z} \mathfrak{F} \leq \mathfrak{F} \mathfrak{A}$, each proper subgroup of $G$ is in $\mathfrak{F} \mathfrak{U} \cup \mathfrak{C}$ and hence $G^{\prime}$ is finite by Corollary 4.6. On the other hand, all proper subgroups of $G$ are obviously abelian-by-finite and so $G$ itself is abelian-by-finite (see, for instance, [15], Lemma 3.1 and Lemma 3.2). Therefore $G / \zeta(G)$ is finite and this contradiction shows that the class $\mathbf{Z} \mathfrak{F} \cup \mathfrak{C}$ is accessible, whence $\mathbf{A}(\mathbf{Z} \mathfrak{F})=\mathbf{Z} \mathfrak{F} \cup \mathfrak{S}$

Let $k$ be an element of the set $\mathbb{N} \cup\{\infty\}$. If $G$ is any group, the subgroup $G_{k}$ generated by all elements of $G$ of order $k$ is called the k-layer of $G$. Clearly, every layer of $G$ is a characteristic subgroup and $G$ is covered by its layers. A group $G$ is called an FL-group if every layer of $G$ is finite, while it is called a $C L$-group if all its layers are Černikov. Thus any $F L$-group is covered by finite normal subgroups and so it is a periodic $F C$-group, while any 
$C L$-group has a covering consisting of Černikov normal subgroups and hence it is locally finite. Actually, it was proved by Polovickii that a group has the $C L$-property if and only if it is covered by Černikov normal subgroups and all its Sylow subgroups are Černikov (see [28] Part 1, Theorem 4.42).

Lemma 4.8 The class of CL-groups is accessible.

Proof Assume for a contradiction that the class of $C L$-groups admits a locally graded opponent $G$, which of course must be locally finite. If $p$ is any prime number and $P$ is any Sylow $p$-subgroup of $G$, it follows from Polovickiı̈'s description of $C L$-groups that all proper subgroups of $P$ are Černikov and hence $P$ itself is a Černikov group. Therefore all Sylow subgroups of $G$ are Černikov and hence $G$ cannot be covered by Černikov normal subgroups, again by the result of Polovickil. On the other hand, every proper subgroup of $G$ has a covering consisting of Černikov normal subgroups and hence it follows from the theorem in [21] that $G$ is a $q$-group for some prime $q$, which is an evident contradiction. Therefore the class of $C L$-groups is accessible.

Since the locally dihedral 2-group contains infinitely many elements of order 2 , it is an opponent of the class of $F L$-groups and so this class is inaccessible, in contrast to the above result.

Corollary 4.9 The accessible closure of the class FL of groups with finite layers is FLU $\mathfrak{C}$.

Proof By Theorem 4.1 we have $\mathfrak{S}=\mathbf{A}(\mathfrak{F}) \leq \mathbf{A}(F L)$, and so $F L \cup \mathfrak{C} \leq \mathbf{A}(F L)$. Assume for a contradiction that there exists a locally graded opponent $G$ of $F L \cup \mathfrak{C}$. Then $G$ is a $C L-$ group by Lemma 4.8, so there exists a positive integer $k$ such that the $k$-th layer $G_{k}$ is an infinite Černikov group. It follows that every proper subgroup of $G / G_{k}$ is Černikov, so that $G$ itself is a Černikov group, a contradiction. Therefore the class $F L \cup \mathfrak{C}$ is accessible and hence $\mathbf{A}(F L)=F L \cup \mathfrak{c}$.

In the last part of this section we describe some examples of accessible closures involving the class $\mathfrak{Q}$ of linear groups. It is well known that locally nilpotent (locally supersoluble) linear groups are hypercentral (hypercyclic) and so it follows from the accessibility of the classes $\mathbf{L} \mathfrak{N}$ and $\mathbf{L}\left(\mathbf{P}_{n} \mathscr{C}\right)$ that

$$
\mathbf{A}(\boldsymbol{Z}) \cap \mathfrak{Q}=\boldsymbol{Z} \cap \mathfrak{L} \quad \text { and } \quad \mathbf{A}\left(\dot{\mathbf{P}}_{n} \mathscr{C}\right) \cap \mathfrak{L}=\dot{\mathbf{P}}_{n} \mathscr{C} \cap \mathfrak{Q} .
$$

Recall now that a group $G$ is paranilpotent if it has a normal series of finite length whose factors are abelian and have only $G$-invariant subgroups. Paranilpotent groups form a class $\mathscr{P}$ that lies strictly between $\mathbf{P}_{n} \mathscr{C}$ and $\dot{\mathbf{P}}_{n} \mathscr{C}$.

Theorem 4.10 $\mathbf{A}(\mathscr{P}) \cap \mathfrak{L}=(\mathscr{P} \cap \mathfrak{Q}) \cup\left(\mathfrak{C} \cap \mathbf{L}\left(\mathbf{P}_{n}^{\mathscr{C}}\right)\right)$.

Proof Obviously, $\mathscr{P} \cap \mathfrak{Q} \leq \mathbf{A}(\mathscr{P}) \cap \mathfrak{Q}$. Moreover, by Lemma 3.7 and Theorem 4.1 we have

$$
\mathfrak{S} \cap \mathbf{L}\left(\mathbf{P}_{n}^{\mathscr{C}}\right)=\mathbf{A}\left(\mathfrak{F} \cap \mathbf{L}\left(\mathbf{P}_{n}^{\mathscr{C}} \mathscr{C}\right) \leq \mathbf{A}(\mathscr{P}) \cap \mathfrak{L} .\right.
$$

Assume for a contradiction $\mathscr{V}=(\mathscr{P} \cap \mathfrak{Q}) \cup\left(\mathfrak{C} \cap \mathbf{L}\left(\mathbf{P}_{n} \mathscr{C}\right)\right)<\mathbf{A}(\mathscr{P}) \cap \mathfrak{Q}$ and let $\mu$ be the least ordinal such that $\mathbf{A}_{\mu}(\mathscr{P}) \backslash \mathscr{V}$ contains a linear group $G$. Then $\mu>0$ is not limit and 
hence $G$ is an opponent of $\mathbf{A}_{\mu-1}(\mathscr{P})$. On the other hand, $\mathbf{A}_{\mu-1}(\mathscr{P}) \cap \mathfrak{Q} \leq \mathscr{V}$ by the minimal choice of $\mu$, so all proper subgroups of $G$ are either paranilpotent or Černikov and hence $G$ is a Černikov group (see [17], Corollary 4.4). Since the class of locally supersoluble groups is accessible, we have that $G$ is locally supersoluble, a contradiction.

Our final result deals with the accessible closure of the class $\mathbf{Z}_{k} \mathfrak{F}$ consisting of all groups which are finite over the $k$-th term of their upper central series for a fixed nonnegative integer $k$.

Theorem 4.11 $\mathbf{A}\left(\mathbf{Z}_{k} \mathfrak{F} \cap \mathfrak{Q}\right)=\left(\mathbf{Z}_{k} \mathfrak{F} \cap \mathfrak{Q}\right) \cup \mathfrak{C}$

Proof By Theorem 4.1 we have $\left(\mathbf{Z}_{k} \mathfrak{F} \cap \mathfrak{Q}\right) \cup \mathfrak{C} \leq \mathbf{A}\left(\mathbf{Z}_{k} \mathfrak{F} \cap \mathfrak{Q}\right)$. Assume for a contradiction that the class $\left(\mathbf{Z}_{k} \mathfrak{F} \cap \mathfrak{Q}\right) \cup \mathfrak{C}$ admits a locally graded opponent $G$. Since $\mathbf{Z}_{k} \mathfrak{F} \leq \mathfrak{F}_{k}$, it follows from Corollary 4.6 that $\gamma_{k+1}(G)$ is finite and hence $G$ is linear (see [19]). On the other hand, it is well known that $\mathfrak{F}_{k} \cap \mathfrak{Q} \leq \mathbf{Z}_{k} \mathfrak{F}$ (see, for instance, [18]). This contradiction completes the proof.

Funding Open access funding provided by Università degli Studi di Napoli Federico II within the CRUICARE Agreement.

Open Access This article is licensed under a Creative Commons Attribution 4.0 International License, which permits use, sharing, adaptation, distribution and reproduction in any medium or format, as long as you give appropriate credit to the original author(s) and the source, provide a link to the Creative Commons licence, and indicate if changes were made. The images or other third party material in this article are included in the article's Creative Commons licence, unless indicated otherwise in a credit line to the material. If material is not included in the article's Creative Commons licence and your intended use is not permitted by statutory regulation or exceeds the permitted use, you will need to obtain permission directly from the copyright holder. To view a copy of this licence, visit http://creativecommons.org/licenses/by/4.0/.

\section{References}

1. Baer, R.: Überauflösbare Gruppen. Abh. Math. Sem. Univ. Hamburg 23, 11-28 (1957)

2. Bruno, B.: On groups with $<<$ abelianbyfinite $>>$ proper subgroups. Boll. Un. Mat. Ital. 3, 797-807 (1984)

3. Bruno, B., Phillips, R.E.: Groups with restricted non-normal subgroups. Math. Z. 176, 199-221 (1981)

4. Bruno, B., Phillips, R.E.: On minimal conditions related to Miller-Moreno type groups. Rend. Sem. Mat. Univ. Padova 69, 153-168 (1983)

5. De Falco, M., de Giovanni, F., Musella, C.: Groups whose finite homomorphic images are metahamiltonian. Comm. Algebra 37, 2468-2476 (2009)

6. Dixon, M.R., Evans, M.J.: Groups with the minimum condition on insoluble subgroups. Arch. Math. (Basel) 72, 241-251 (1999)

7. Dixon, M.R., Evans, M.J., Smith, H.: Groups with some minimal conditions on non-nilpotent subgroups. J. Group Theory 4, 207-215 (2001)

8. Dixon, M.R., Evans, M.J., Smith, H.: Groups with all proper subgroups soluble-by-finite rank. J. Algebra 289, 135-147 (2005)

9. Dixon, M.R., Ferrara, M., Karatas, Z.Y., Trombetti, M.: Groups with all subgroups permutable or soluble of finite rank. J. Algebra 549, 195-214 (2020)

10. Doerk, K.: Minimal nicht überauflösbare, endliche Gruppen. Math. Z. 91, 198-205 (1966)

11. Ferrara, M., Trombetti, M.: A local study of group classes. Note Mat. 42, 1-20 (2020)

12. Franciosi, S., de Giovanni, F., Newell, M.L.: Groups with polycyclic non-normal subgroups. Algebra Colloq. 7, 33-42 (2000) 
13. de Giovanni, F., Trombetti, M.: Infinite minimal non-hypercyclic groups. J. Algebra Appl. 14(10), 1550143 (2015)

14. de Giovanni, F., Trombetti, M.: Countable recognizability and nilpotency properties of groups. Rend. Circ. Mat. Palermo 66, 399-412 (2017)

15. de Giovanni, F., Trombetti, M.: Groups whose proper subgroups are metahamiltonian-by-finite. Rocky Mountain J. Math. 50, 153-162 (2020)

16. de Giovanni, F., Trombetti, M.: "Cohopfian groups and accessible group classes", Pacific J. Math., to appear

17. de Giovanni, F., Trombetti, M., Wehrfritz, B.A.F.: Linear groups whose proper subgroups are close to being nilpotent. Comm. Algebra 49, 3020-3033 (2021)

18. de Giovanni, F., Trombetti, M., Wehrfritz, B.A.F.: "The upper and lower central series in linear groups", Quart. J. Math., to appear

19. de Giovanni, F., Trombetti, M., Wehrfritz, B.A.F.: "Groups whose proper subgroups are linear", to appear

20. Hartley, B.: Finite groups of automorphisms of locally soluble groups. J. Algebra 57, 242-257 (1979)

21. Hartley, B., Otal, J., Peña, J.M.: Locally graded minimal non-CC groups are p-groups. Arch. Math. (Basel) 57, 209-211 (1991)

22. Heineken, H., Mohamed, I.J.: A group with trivial centre satisfying the normalizer condition. J. Algebra 10, 368-376 (1968)

23. Kleidman, P.B., Wilson, R.A.: A characterization of some locally finite simple groups of Lie type. Arch. Math. (Basel) 48, 10-14 (1987)

24. Kuzucuoğlu, M., Phillips, R.E.: Locally finite minimal non-FC-groups. Math. Proc. Cambridge Philos. Soc. 105, 417-420 (1989)

25. Morse, A.P.: A Theory of Sets. Academic Press, Orlando (1986)

26. Otal, J., Peña, J.M.: Infinite locally finite groups of type $P S L(2, K)$ or $S z(K)$ are not minimal under certain conditions. Pub. Mat. 32, 43-47 (1988)

27. Phillips, R.E., Wilson, J.S.: On certain minimal conditions for infinite groups. J. Algebra 51, 41-68 (1978)

28. Robinson, D.J.S.: Finiteness Conditions and Generalized Soluble Groups. Springer, Berlin (1972)

29. Romalis, G.M., Sesekin, N.F.: Metahamiltonian groups. Ural. Gos. Univ. Mat. Zap. 5, 101-106 (1966)

30. Romalis, G.M., Sesekin, N.F.: Metahamiltonian groups II. Ural. Gos. Univ. Mat. Zap. 6, 50-52 (1968)

31. Romalis, G.M., Sesekin, N.F.: Metahamiltonian groups III. Ural. Gos. Univ. Mat. Zap. 7, 195-199 (1969)

32. Schmidt, R.: Subgroup Lattices of Groups. de Gruyter, Berlin (1994)

33. Smith, G.C.: Compressibility in nilpotent groups. Bull. London Math. Soc. 17, 453-457 (1985)

34. Sunkov, V.P.: The problem of minimality for locally finite groups. Algebra Logic 9, 137-151 (1970)

35. Tomkinson, M.J.: FC-groups. Pitman, Boston (1984)

Publisher's Note Springer Nature remains neutral with regard to jurisdictional claims in published maps and institutional affiliations. 